\title{
Study of the trigger mode of LHAASO-KM2A
}

\section{Sha Wu*}

Key Laboratory of Particle Astrophysics, Institute of High Energy Physics, Chinese Academy of Sciences, 100049 Beijing, P.R. China.

University of Chinese Academy of Sciences, 100049 Beijing, P.R.China.

E-mail: wushadihep.ac.cn

\section{Songzhan Chen}

Key Laboratory of Particle Astrophysics, Institute of High Energy Physics, Chinese Academy of Sciences, 100049 Beijing, P.R. China.

\section{Huihai He}

Key Laboratory of Particle Astrophysics, Institute of High Energy Physics, Chinese Academy of Sciences, 100049 Beijing, P.R. China.

\section{Xiong Zuo ${ }^{\dagger}$}

Key Laboratory of Particle Astrophysics, Institute of High Energy Physics, Chinese Academy of Sciences, 100049 Beijing, P.R. China.

\section{On behalf of the LHAASO Collaboration}

The Large High Altitude Air Shower Observatory (LHAASO), to be built at $4410 \mathrm{~m}$ a.s.l. near the Daocheng in the Sichuan province of China, is a new generation EAS array. It consists of three kinds of detector arrays. KM2A is the main array of LHAASO and is composed of 5195 electromagnetic particle detectors and 1171 muon detectors, which are distributed in an area of $1.3 \mathrm{~km}^{2}$. In this work, we study the trigger logics of KM2A, which will adopt an unprecedented software trigger system. Taking into account the characters of real cosmic ray events, we investigate the trigger logics by adjusting the space window and time window together. In this study, we adopt the CORSIKA to simulate the shower development and a parameterization code to simulate the detector response. We also develop a toy simulation to estimate the noise event rate due to chance coincidence noise hits. With the principle to maximize the real cosmic ray events in the same time to inhibit the noise events, we achieve an optimized trigger criterion for KM2A. The corresponding threshold energy for gamma-ray is about $4 \mathrm{TeV}$, which is much lower than the previous initial trigger logic. The performances of KM2A with this trigger criterion are also presented.

35th International Cosmic Ray Conference - ICRC2017

10-20 July, 2017

Bexco, Busan, Korea

${ }^{*}$ This work is supported in China by NSFC No.11575203,11635011,11605208,11375210

†speaker. 


\section{Introduction}

Cosmic rays with maximum energy up to about $10^{20} \mathrm{eV}$ are the most energetic particles in the universe. Cosmic rays mainly consist of charged nuclei and have been deflected by the Galactic magnetic field in their path propagating to the Earth. This makes it difficult to identify the original sources, even though the cosmic rays with energy up to $10^{15} \mathrm{eV}$ are believed to originate from Galactic compact objects, e.g., supernova remnant. Therefore, the origin of cosmic rays is recognized as one of the most important puzzles in the field of particle astrophysics. The neutral high energy gamma-rays, produced by collision between cosmic ray hadrons and the ambient mediums, are important probe to detect the smoke gun of cosmic ray origination.

The Large High Altitude Air Shower Observatory (LHAASO) project [U, ] is a complex extensive air shower (EAS) array and will be built at Haizi Mountain (4410 m a.s.l.) in Sichuan province, China. One of the most important science motivations is to extend the current very high energy (VHE) gamma-ray observation from about $0.1-20 \mathrm{TeV}$ to energy about $100 \mathrm{TeV}$, which is implemented by a sub array of KM2A of LHAASO. KM2A is composed of more than five thousand particle detectors. An EAS, composed of many secondary particles, can fire multiple detectors (one fire will be denoted as one hit in the following text) in a short time period. The number of hits is energy dependent. While one detector can also be fired by random signal particle and multiple detectors have a certain possibility to be fired coincidentally in a short period (this will be denoted as noise event in the following text). Therefore, a trigger logic is need to reject noise events and select real EAS events.

The trigger system of KM2A will fully adopt an unprecedented software trigger system. This will allow a complex trigger logics to select events as lowest energy as possible basing on shower topology. In this paper, we will present the study of trigger logics of KM2A.

\section{The KM2A detector and simulation}

\subsection{The KM2A detector}

LHAASO is a major instrument for VHE gamma-ray astronomy and cosmic ray physics. It is a complex EAS array and consists of three sub-arrays, i.e., $1 \mathrm{~km}^{2}$ array (KM2A), 78,000 $\mathrm{m}^{2}$ water Cherenkov detector array (WCDA), and wide-field air Cherenkov telescope array (WFCTA). The

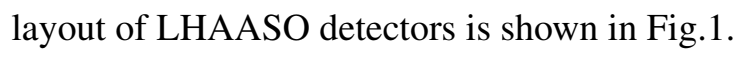

As the major array of LHAASO, KM2A is comprised of 5195 Electromagnetic particle Detector (EDs) and 1171 muon detectors (MDs). ED mainly detects the electromagnetic particles in the shower, which will be used to reconstruct the primary direction, core location and energy. In the following study, concerning the trigger logic, only the EDs are taken into account. To test the KM2A design, a porotype engineer array about $1 \%$ of KM2A was constructed at yangbajing, Tibet, China [B]. The resulting performances of ED fully meet the design requirements. More details about the ED and its performances can be found elsewhere [ [ 9 , []. According to the engineer array result, the noise rate is $2 \mathrm{kHz}$ for each ED. Two prototype MDs were constructed in the engineer array in 2012 and 2014, respectively. More details about the MD and its performances can be found elsewhere [目]. According to the prototype MD result, the noise rate is $6 \mathrm{kHz}$. 


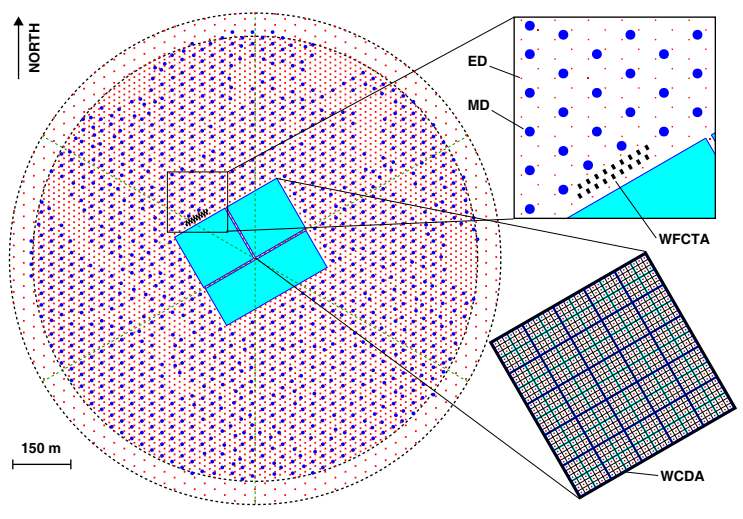

Figure 1: The layout of LHAASO detectors

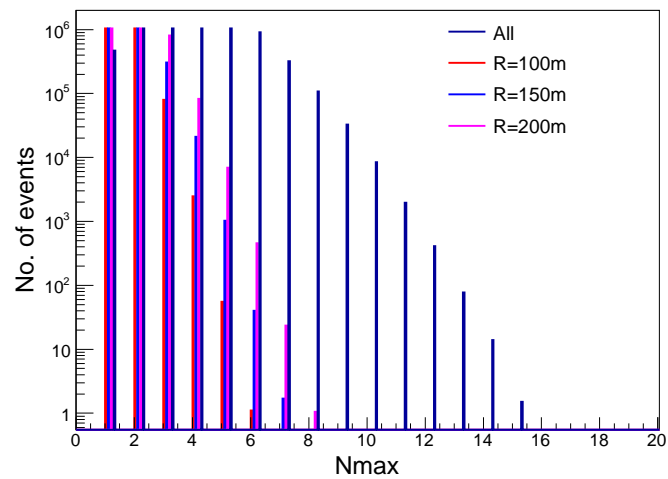

Figure 2: The distribution of $\mathrm{N}_{\max }$ for $\mathrm{T}_{\text {wind }}=200 \mathrm{~ns}$. Different lines stand for $\mathrm{R}_{\text {wind }}=100 \mathrm{~m}, 150 \mathrm{~m}, 200 \mathrm{~m}$, and infinite, respectively. The values of $\mathrm{N}_{\max }$ have been slightly shifted for separation.

\subsection{Detector simulation}

The EAS process is simulated by Corsika version 7.4005. The energy of primary gamma-ray and nuclei are sampled within $1-1000 \mathrm{TeV}$ following a simple power law function. The zenith angles are sampled within $0^{\circ}-60^{\circ}$. For detector response, a parameterized method presented in [0] is adopted here. That is, the ED and MD detector unit of the KM2A array are simulated with Geant4 individually at first. Then a look-up table is made to parameterize the number of generated particles corresponding to different injected particles. One particle is parameterized to photo-electrons which is sampled with Landau distribution according to the real signal recorded by the KM2A engineering array [B]]. Besides these, random noises from single secondary cosmic ray in the EDs and MDs are also taken into account with noise rates of $2 \mathrm{kHz}$ and $6 \mathrm{kHz}$ for ED and $\mathrm{MD}$, respectively. Finally, these signals are digitized to hits according the electronic performances used in the KM2A engineering array. For ED, the time resolution is set to be $2 \mathrm{~ns}$ with a dead time of $8 \mathrm{~ns}$ for each hit. For MD, the time resolution is set to be $10 \mathrm{~ns}$ with a dead time of $600 \mathrm{~ns}$. The cores are sampled within a large enough circular area region with radius of $1000 \mathrm{~m}$ centering on KM2A.

\section{Method}

When a VHE gamma-ray (or cosmic ray nuclei) enters the atmosphere, it interacts with atmospheric nuclei and induces electromagnetic cascades, i.e., an EAS. Most of the secondary particles are distributed in a large disk, few meters thick and orthogonal to the direction of primary particle. For the detectors on the observation plane, the spread of arrival time is dependent on the primary zenith angle. The lateral extent of secondary particles is about hundreds meters. The particle density is significantly correlated with the distance to the shower core. Therefore, besides using the time window, the space window is also adopted here to study the trigger logic. In this work, three time windows (denoted as $\mathrm{T}_{\text {wind }}$ ), i.e., $200 \mathrm{~ns}, 300 \mathrm{~ns}$, and $400 \mathrm{~ns}$, and four circular space windows (denoted as $\mathrm{R}_{\text {wind }}$ ), i.e., with the radius of $100 \mathrm{~m}, 150 \mathrm{~m}, 200 \mathrm{~m}$, and infinite, are investigated. The 
threshold, i.e., the minimum of fired EDs (denoted as $N_{t}$ ), for a specific time and space window is mainly determined by the noise event rate due to random coincident.

\subsection{Estimation of noise event rate}

According to KM2A engineering array, the noise hit rate of one ED is $2 \mathrm{kHz}$. For the whole $5195 \mathrm{EDs}$, there is about one hit in every $100 \mathrm{~ns}$ on average. In the real experiment, the trigger system will monitor the hits one by one and the trigger logic will be implement about $10^{7}$ times per second. For such a large test number, the noise hits will have a high possibility to be random coincident. To estimate the trigger rate due to random coincident, a toy Monte Carlo (MC) simulation method is adopted.

In this toy MC, one ED is randomly selected as the first fired ED. In the following time window $\mathrm{T}_{\text {wind }}$, the number of fired EDs is sampled using a Poisson distribution with the expected mean value of $\mathrm{T}_{\text {wind }} \times 2000 \mathrm{~Hz} \times 5195$. The fired EDs are randomly sampled in the whole EDs. Then, the maximum number of fired $\mathrm{ED}$ (denoted as $\mathrm{N}_{\max }$ ) within a circular space window with radius of $\mathrm{R}_{\text {wind }}$ is searched by centering the fired EDs one by one. Finally, the above procedures are repeated $2 \times 10^{8}$ times, corresponding to $20 \mathrm{~s}$ in total. With the distribution of $\mathrm{N}_{\max }$, the noise event rate due to random coincident under different threshold $\mathrm{N}_{t}$ can be estimated. $\mathrm{N}_{t}$ is defined as the minimum number of fired detector required for a trigger logic. As an example, Fig. $\square$ shows the distribution of $\mathrm{N}_{\text {max }}$ for $\mathrm{T}_{\text {wind }}=200 \mathrm{~ns}$ with different $\mathrm{R}_{\text {wind }}$. As shown in the Figure, the random coincident can be significantly reduced with tightening the space windows.

\subsection{Optimization Strategy}

The main goal of a trigger logic is to select the real EAS events as more as possible in the same time inhibiting the noise events. In this work, some strategies are used in optimizing the trigger logic. (1) The noise event rate due to random coincident should be less than $10 \mathrm{~Hz}$, which is an arbitrary decision and will be discussed later. (2) The threshold $\mathrm{N}_{t}$ should be greater than 4 , which is the number of free parameters in the direction reconstruction. (3) the real EAS events should survive the trigger logic as more as possible. The situation is much more complicated for different trigger logics if taking into account the noise hits. Therefore, the noise hits are not taken into account in detector simulation when study the utility of trigger logic for EAS.

\section{Result}

\subsection{Noise event rate and threshold}

Using the toy MC described in last section, the noise event rates due to random coincident under different threshold $\mathrm{N}_{t}$ are estimated. The noise event rates as the function of $\mathrm{N}_{t}$ for different $\mathrm{T}_{\text {wind }}$ and $\mathrm{R}_{\text {wind }}$ are shown in Fig. 3 . According to the optimization strategy that the noise event rate should be less than $10 \mathrm{~Hz}$, the require minimum threshold $\mathrm{N}_{t}$ can be obtained basing on the result presented in Fig. [3. The derived $\mathrm{N}_{t}$ for different time and space windows are summarized in Table m.If only the time window is used, i.e., the space window is infinite, the threshold $\mathrm{N}_{t}$ is $15,16,20$, for $\mathrm{T}_{\text {wind }}=200 \mathrm{~ns}, 300 \mathrm{~ns}, 400 \mathrm{~ns}$, respectively. If the spacing window is also used, the threshold $\mathrm{N}_{t}$ can decrease to 6. With these thresholds for different windows, the utility for EAS is investigated. 

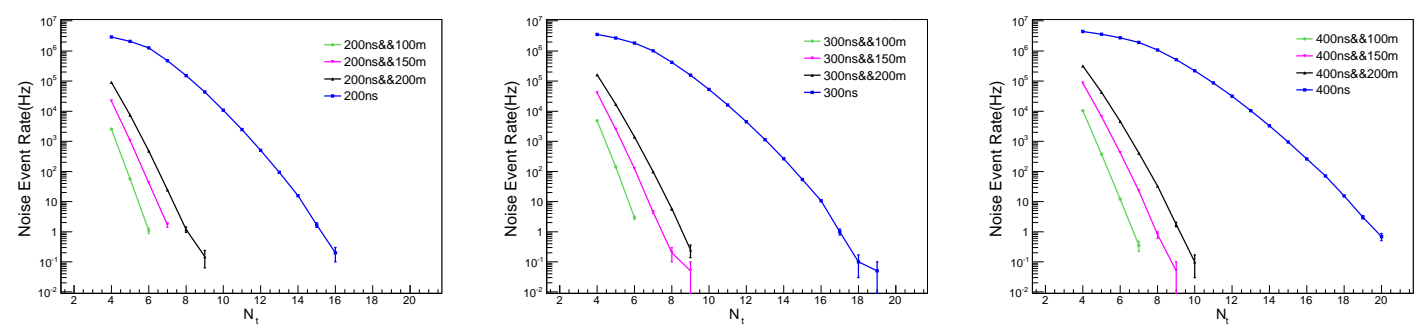

Figure 3: The noise event rates as the function of $\mathrm{N}_{t}$ for $\mathrm{T}_{\text {wind }}=200 \mathrm{~ns}$ (Left), $300 \mathrm{~ns}$ (Middle), $400 \mathrm{~ns}$ (Right). Different curves stand for $\mathrm{R}_{\text {wind }}=100 \mathrm{~m}, 150 \mathrm{~m}, 200 \mathrm{~m}$, and infinite, respectively.

\subsection{Optimal trigger logic for EAS}

To compare the utility of different trigger logics for EAS, the detector simulation procedures described in section 2.2 are used, while, the noise hits are not taken into account as interpreted in section 3.2. For this study, the gamma-rays with primary energy from $1 \mathrm{TeV}$ to $1000 \mathrm{TeV}$ are used. The spectrum follow a simple power-law function with spectral index of -2.5. The result would be similar if using cosmic ray such as Proton.

The distributions of gamma-ray energies that survive the trigger criterions listed in Table $\square$ are shown in Fig.因. According to Fig. 田, the utility of different trigger criterions is very similar at high energy. The main differences appear in the low energy. The tendency is that smaller window can select more shower events to extend to lower energy. For quantification, the number of triggered gamma-rays are also summarized in Table $\mathbb{W}$ which have been normalized to the event number of $\mathrm{T}_{\text {wind }}=200 \mathrm{~ns}$ and $\mathrm{R}_{\text {wind }}=100 \mathrm{~m}$.
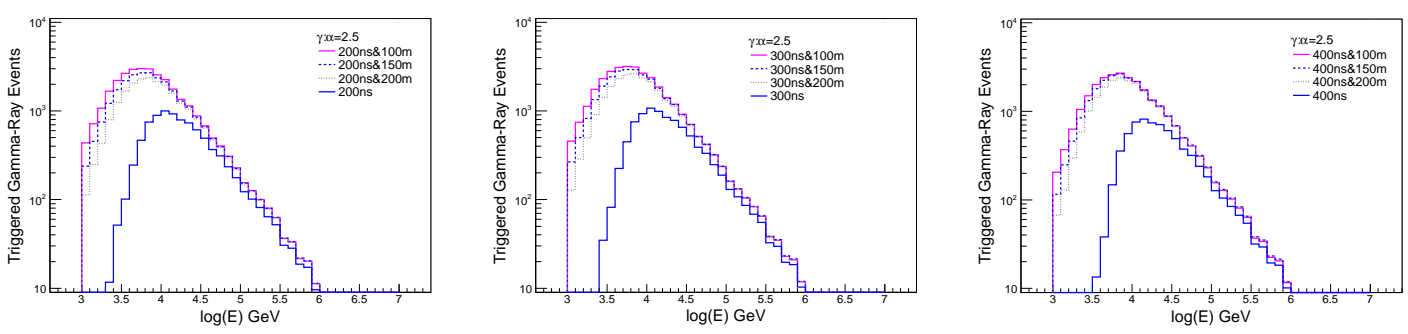

Figure 4: Distribution of gamma-ray energies that survive the different trigger criterions listed in Table $\mathbb{W}$.

According to the result presented in Table $\square$ and Fig. 9 , if only the time window is used, the best trigger criterion is $\mathrm{T}_{\text {wind }}=300 \mathrm{~ns}$ and $\mathrm{N}_{t}=16$, which will select $26 \%$ more events than using $\mathrm{T}_{\text {wind }}=400 \mathrm{~ns}$ and $\mathrm{N}_{t}=20$. If the space window is also used, the best trigger criterion would be $\mathrm{T}_{\text {wind }}=300 \mathrm{~ns}, \mathrm{R}_{\text {wind }}=100 \mathrm{~m}$ and $\mathrm{N}_{t}=6$. This criterion will select $262 \%$ more events that only using time window, i.e., $\mathrm{T}_{\text {wind }}=300 \mathrm{~ns}$ and $\mathrm{N}_{t}=16$. The corresponding threshold energy for gamma-ray also decreases as shown in Fig.⿴囗十.

\section{Discussion}

According to the previous result, the trigger criterion of $\mathrm{T}_{\text {wind }}=300 \mathrm{~ns}, \mathrm{R}_{\text {wind }}=100 \mathrm{~m}$ and $\mathrm{N}_{t}=6$ can select more EAS than others. To check how many EAS are rejected by this trigger criterion, All 


\begin{tabular}{ccccc}
\hline $\mathrm{T}_{\text {wind }}$ & $\mathrm{R}_{\text {wind }}$ & $\mathrm{N}_{t}$ & Noise event Rate $(\mathrm{Hz})$ & normalized gamma-ray event \\
\hline $200 \mathrm{~ns}$ & $100 \mathrm{~m}$ & 6 & $1.10 \pm 0.23$ & 1 \\
& $150 \mathrm{~m}$ & 7 & $1.69 \pm 0.29$ & 0.87 \\
& $200 \mathrm{~m}$ & 8 & $1.19 \pm 0.24$ & 0.74 \\
& $\mathrm{No}$ & 15 & $1.69 \pm 0.29$ & 0.28 \\
\hline $300 \mathrm{~ns}$ & $100 \mathrm{~m}$ & 6 & $2.95 \pm 0.38$ & 1.05 \\
& $150 \mathrm{~m}$ & 7 & $4.45 \pm 0.47$ & 0.95 \\
& $200 \mathrm{~m}$ & 8 & $5.79 \pm 0.54$ & 0.82 \\
& $\mathrm{No}$ & 16 & $10.0 \pm 0.73$ & 0.29 \\
\hline $400 \mathrm{~ns}$ & $100 \mathrm{~m}$ & 7 & $0.55 \pm 0.17$ & 0.85 \\
& $150 \mathrm{~m}$ & 8 & $1.95 \pm 0.31$ & 0.81 \\
& $200 \mathrm{~m}$ & 9 & $6.20 \pm 0.56$ & 0.72 \\
& No & 20 & $3.29 \pm 0.41$ & 0.23 \\
\hline
\end{tabular}

Table 1: The trigger criterions, the noise event rates and survival gamma-rays.

the showers that can fired more than 4 EDs in the previous simulation are investigated. As shown in Fig.[1, the superiority of trigger criterion for $\mathrm{T}_{\text {wind }}=300 \mathrm{~ns}$ and $\mathrm{R}_{\text {wind }}=100 \mathrm{~m}$ is clear compared to the trigger criterion for $\mathrm{T}_{\text {wind }}=300 \mathrm{~ns}$. All the rejected showers are small showers. More than $95 \%$ showers with $\mathrm{N}_{E D}>10$ can survive the trigger criterion for $\mathrm{T}_{\text {wind }}=300 \mathrm{~ns}$ and $\mathrm{R}_{\text {wind }}=100 \mathrm{~m}$. Therefore, the utility of current trigger logic preform well in select real showers.

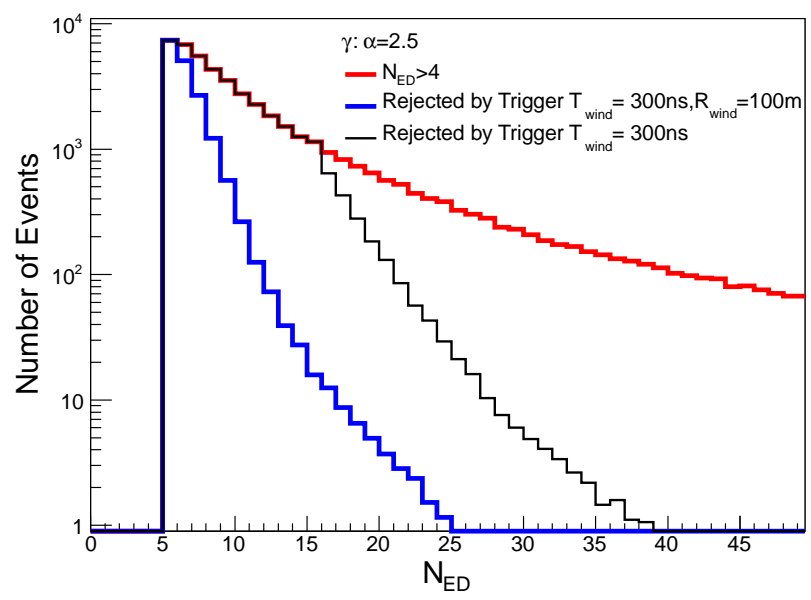

Figure 5: Distribution of $\mathrm{N}_{E D}$ for all showers with $\mathrm{N}_{E D}>4$ and the showers that rejected by two trigger criterions.

In the primary optimization strategy, an arbitrary decision is adopted to limit the noise event rate due to random coincident less than $10 \mathrm{~Hz}$. To investigate the influence of this limitation, the threshold $\mathrm{N}_{t}$ for different windows is set to be 6 . The noise event rate and the number of survival gamma-rays using different trigger criterions are estimated and summarized in Table $\square$. As listed in Table $\square$, when the noise event rate increases from a few $\mathrm{Hz}$ to hundreds $\mathrm{Hz}$, the fraction of selected gamma-ray showers only increases by a factor of about $10 \%$. According to the result shown in 


\begin{tabular}{cccc}
\hline $\mathrm{T}_{\text {wind }}$ & $\mathrm{R}_{\text {wind }}$ & Noise event Rate $(\mathrm{Hz})$ & normalized gamma-ray event \\
$200 \mathrm{~ns}$ & $100 \mathrm{~m}$ & $1 \pm 0.23$ & 1 \\
& $150 \mathrm{~m}$ & $38 \pm 1.37$ & 1.09 \\
& $200 \mathrm{~m}$ & $437 \pm 4.67$ & 1.13 \\
\hline $300 \mathrm{~ns}$ & $100 \mathrm{~m}$ & $3 \pm 0.37$ & 1.05 \\
& $150 \mathrm{~m}$ & $124 \pm 2.49$ & 1.18 \\
& $200 \mathrm{~m}$ & $1270 \pm 7.97$ & 1.24 \\
\hline $400 \mathrm{~ns}$ & $100 \mathrm{~m}$ & $22 \pm 1.03$ & 1.05 \\
& $150 \mathrm{~m}$ & $854 \pm 6.53$ & 1.21 \\
& $200 \mathrm{~m}$ & $94503 \pm 21.7$ & 1.28 \\
\hline
\end{tabular}

Table 2: The noise event rate and survival gamma-rays of different trigger criterions with $\mathrm{N}_{t}=6$. For the number of triggered gamma-rays, the same normalization as that in Table $\square$ is applied.

Fig.5, the additional events should be small showers with $\mathrm{N}_{E D} \leq 13$. For such a situation, the primary optimization strategy is reasonable to limit the noise event rate less than $10 \mathrm{~Hz}$.

To check the performance of KM2A using the trigger logic obtained in this work,the effective area is shown in Fig.6. In this study, the noise hits of each ED are taken into account, which will increases the trigger efficiency more or less and the sample area is $3.14 \mathrm{~km}^{2}$ as stated in Section 2.2. The effective area is about $0.98 \mathrm{~km}^{2}$ at $10 \mathrm{TeV}$ and $1.63 \mathrm{~km}^{2}$ at $100 \mathrm{TeV}$. For comparison, the effective area using the trigger criterion for $\mathrm{T}_{\text {wind }}=300 \mathrm{~ns}$ are also shown in Fig.6. To check the trigger efficiency for the showers with core located in the central area of KM2A (denoted as internal showers), the effective area for internal showers are estimated and also shown in Fig.6. As shown, it approach to full efficiency for gamma-ray with energy above $100 \mathrm{TeV}$.
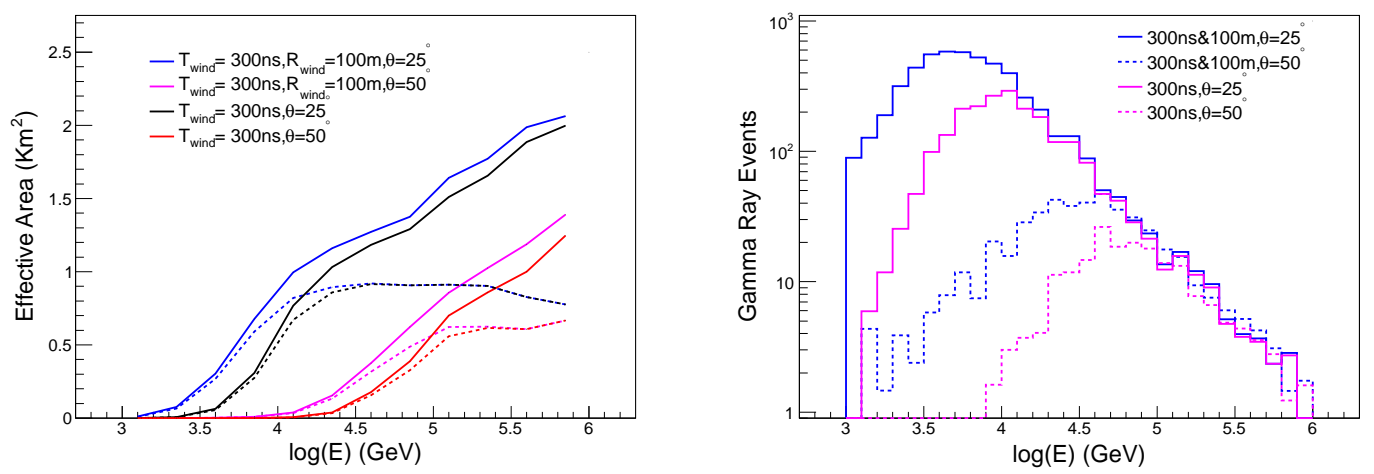

Figure 6: KM2A effective areas for gamma-rays as Figure 7: KM2A response to the gamma-ray speca function of the energy for two trigger criterions. trum with $\alpha=-2.5$ using two trigger criterions. The The solid lines are obtained with all the triggered zenith angles are $\theta=25^{\circ}$ and $\theta=50^{\circ}$. events. The dotted lines stands for the events with primary core located in KM2A center area and the same color stands for the same trigger condition.

KM2A response to the gamma-ray spectrum can be estimated by multiplying with the effective area . Fig.D shows the response for gamma-ray spectrum with a simple power-law function with 
$\alpha=-2.5$. The zenith angles are $\theta=25^{\circ}$ and $\theta=50^{\circ}$. The threshold energy is about $3.2 \mathrm{TeV}$ and 32 $\mathrm{TeV}$ for $\theta=25^{\circ}$ and $\theta=50^{\circ}$, respectively. For comparison, the energy distribution using trigger criterion for $\mathrm{T}_{\text {wind }}=300 \mathrm{~ns}$ are also shown in Fig.D, which shows a threshold energy about $10 \mathrm{TeV}$ and $56 \mathrm{TeV}$, respectively. It is clear that the threshold energy can be reduced after adding the space window.

The flux of nearly isotropy hadronic cosmic ray is much higher than that of gamma-ray. The trigger events of KM2A will be dominated by hadronic cosmic ray in the future. To estimate the trigger rate of KM2A using the trigger logic obtained in this work, the phenomenological model about cosmic ray composition and the corresponding spectra described in [ $[\mathrm{B}]$ is adopted. The estimated trigger rate is around $6.3 \mathrm{kHz}$ of the best trigger criterion. The trigger rate, using trigger criterion for $\mathrm{T}_{\text {wind }}=300 \mathrm{~ns}$, is around $2.7 \mathrm{kHz}$.

\section{Summary}

The LHAASO is a new generation EAS array and the construction is expected to be finished in 2020. KM2A is the main array of LHAASO for gamma-ray astronomy and cosmic ray physics at energy above $20 \mathrm{TeV}$. In this work, we study the trigger logics of KM2A with different time and space windows. The achieved best trigger logic is that with time window of $300 \mathrm{~ns}$, space window with radius of $100 \mathrm{~m}$, and threshold of 6 fired EDs. This trigger logic have a high efficiency to select real shower event. With this trigger logic, the noise event rate is $3 \mathrm{~Hz}$ and the trigger rate due to cosmic ray showers is about $6.3 \mathrm{kHz}$. The threshold energy for gamma-ray is about $4 \mathrm{TeV}$, which is lower than the initial design, i.e., $20 \mathrm{TeV}$. Such an improvement provides a potential to improve the sensitivity of LHAASO at energies of 5-20 TeV, which is the connecting energy range between KM2A and WCDA.

\section{References}

[1] Cao Z. A future project at tibet: the large high altitude air shower observatory (LHAASO)*, Chinese Physics C, 2010, 34:249

[2] He H. H.Proc. ICRC, 2015, 236:1010

[3] Liu J., et al. Performances and long-term stability of the LHAASO-KM2A prototype array, Chinese Physics C, 2014, 38:40-44

[4] Zhao J., et al. Design and performances of electromagnetic particle detector for LHAASO-KM2A, Chinese Physics C, 2014, 38:31-38

[5] Zhang Z.Q., et al. Study on the performance of electromagnetic particle detectors of LHAASO-KM2A, Nuclear Instruments and Methods in Physics Research A, 2017, 845:429íC433

[6] Zuo X., et al. Design and performances of prototype muon detectors of LHAASO-KM2A, Nuclear Instruments and Methods in Physics Research A, 2015, 789:143íC149

[7] Cui S. W., et al. Simulation on gamma ray astronomy research with LHAASO-KM2A, Astroparticle Physics, 2014, 54:86-92

[8] Gaisser T. K. ařspectrum of cosmic-ray nucleons, kaon production, and the atmospheric muon charge ratio, Astroparticle Physics,2012, 35:801-806 